\title{
Innovations in suicide assessment and prevention during pandemics
}

\section{Connor TA Brenna, ${ }^{a, f}$ Paul S Links ${ }^{b}$, Maxwell M Tranª, Mark Sinyor ${ }^{c}$, Marnin J Heiseld and Simon Hatchere}

a Postgraduate Medical Education, University of Toronto, Ontario, Canada

b Department of Psychiatry and Behavioural Neurosciences, McMaster University, Hamilton, Ontario, Canada

c Sunnybrook Research Institute, University of Toronto, Ontario, Canada

d Lawson Health Research Institute, University of Western Ontario, London, Canada

e Ottawa Hospital Research Institute, University of Ottawa, Ontario, Canada

f Corresponding author: connor.brenna@mail.utoronto.ca

\section{Article history}

Publication date: September 2021

Citation: Brenna CTA, Links PS, Tran MM,

Sinyor M, Heisel MJ, Hatcher S. Innovations in suicide assessment and prevention during pandemics. Public Health Res Pract. 2021;31(3):e3132111. https://doi. org/10.17061/phrp3132111

\section{Key points}

- Emerging evidence suggests that suicide risk (and related behaviours such as self-harm) increased during previous pandemics and may similarly increase during the COVID-19 pandemic

- Certain groups can be identified as particularly vulnerable to suicide risk during pandemics, including older or dependent adults

- There is a critical need for innovations in suicide assessment to mitigate these novel risks

- Pandemic circumstances warrant the uptake and validation of virtual suicide risk assessment tools, including telemedicine, smartphone apps, and internet-based tools, in combination with existing assessment tools and approaches

\section{Abstract}

Emerging evidence, based on the synthesis of reports from past infectious disease-related public health emergencies, supports an association between previous pandemics and a heightened risk of suicide or suicide-related behaviours and outcomes. Anxiety associated with pandemic media reporting appears to be one critical contributing factor. Social isolation, loneliness, and the disconnect that can result from public health strategies during global pandemics also appear to increase suicide risk in vulnerable individuals. Innovative suicide risk assessment and prevention strategies are needed to recognise and adapt to the negative impacts of pandemics on population mental health.

\section{Do pandemics lead to an increase in suicide rates?}

It has been suggested that the coronavirus disease 2019 (COVID-19) pandemic may lead to increased suicide rates. To investigate the potential impact of pandemics on suicide, we systematically reviewed the association between past infectious disease-related public health emergencies (pandemics and epidemics) and suicide or suicide-related outcomes, and assessed the implications of these associations for mental health professionals. A full description of the methodology appears in our original report. ${ }^{1}$ A thorough search of six major databases identified eight primary articles which met the study inclusion criteria: four related to the Severe Acute Respiratory Syndrome (SARS) epidemic, one related to each of the Spanish Flu pandemic, Russian influenza, and ebola virus disease, and the other related to influenza $A$, influenza $B$, and coronaviruses. The eight primary articles were reviewed and, although these were generally of low methodological quality, the available evidence supported an association between previous infectious disease-related public health emergencies and 
an increased risk of suicidal ideation, self-harm, and death by suicide.

Collectively, these studies suggested that certain high-risk groups were the most vulnerable to the suicidogenic effects of pandemics, including older adults and older women in particular; individuals who are or who become unemployed or under-employed; individuals with pre-existing mental health and/or substance misuse problems; and frontline health and social service workers. The authors of this systematic review have engaged in knowledge dissemination regarding the association between pandemics and suicide and have highlighted clinical innovations that may be needed to mitigate risk.

Early data from the COVID-19 pandemic suggest that, although case reports identify individuals who engaged in suicide behaviour or died by suicide in the context of COVID-19 infection or anxiety, suicide and suicide-related behaviours have remained largely stable in high- and upper-middle-income countries ${ }^{2}$, whereas a large population-based cohort study of more than 14 million individuals in the UK reported an initial decrease (followed by a normalisation) in the incidence of self-harm. ${ }^{3}$ Nevertheless, initial stability or decreases in the risk of suicide and suicide-related behaviour during the acute phase of the COVID-19 pandemic may not be predictive of long-term risks, and the emerging reports may be likened to the 'honeymoon period' or 'pulling together' effect previously described in the aftermath of large-scale disasters. ${ }^{1}$ A living systematic review continues to examine the emerging data ${ }^{4}$, which remains limited in its ability to predict the future effects of the COVID-19 pandemic and its immediate aftermath on suicide and related behaviours.

\section{What innovations are needed to reduce suicide risk during the COVID-19 pandemic?}

Our systematic review was constrained by its inclusion of relatively few robust original reports, and yet it supported an association between previous pandemics and increased risk for suicide-related outcomes. Whereas multiple potential mechanisms for this association were proposed, the review found evidence supporting two mechanisms. First, anxiety and dread associated with media reports of the pandemic and its impact on community health appeared to promote the risk of suicide. Second, social isolation and loneliness that resulted from the public health strategies adopted to control the pandemic also appeared to increase suicide risk among vulnerable individuals.

Although the entire population may be affected, at least to some extent, by both of these risk factors, the degree to which they are likely to result in suicide-related outcomes at an individual level is contingent on their interaction with each person's pre-existing vulnerability and specific social contexts. That is, the circumstances of the pandemic may be likely to substantially heighten risk in those prone to anxiety or dread when exposed to mass fear and/or those who have difficulty tolerating isolation and physical distancing. For others it may have no impact, or even reduce risk by virtue of increased time spent at home with family and away from other potential environments of stress.

Mental health professionals should recognise that during infectious disease-related public health emergencies, the population as a whole can be at increased risk for psychological distress. Specific individuals may be especially vulnerable and therefore warrant additional attention and resources, such as outreach and support for older adults who are totally or partially dependent, or those with pre-existing health anxiety. Care providers must be aware of potential social, psychological, and environmental antecedents that may negatively impact mental health and increase risk of suicide. Innovative interventions will be needed for those identified as potentially vulnerable or at risk, including social outreach, virtual suicide risk screening, assessment, and targeted prevention strategies.

In terms of prevention based on the plausible mechanisms identified through the systematic review, clinicians should select from a number of strategies which could be employed with their patients. Vulnerable individuals should be counselled to limit their own exposure to media reports about the pandemic if engagement with media is causing significant distress. Furthermore, attempts should be made to increase social support for vulnerable individuals, particularly older adults, by promoting interpersonal connections with family members, friends and neighbours, as well as community-based social supports and telephone befriending or distress lines, employing in-person and virtual means in accordance with necessary local public health recommendations.

\section{How should virtual suicide risk assessments and crisis management be carried out?}

The unique circumstances of the pandemic require the use of virtual and/or telehealth suicide risk assessment approaches. Stanley and Brown have developed a telehealth tips handout, 'Managing suicidal clients during the COVID-19 pandemic', which is freely available online ${ }^{5}$ and can be easily adopted by care providers undertaking virtual suicide risk assessments. Other online resources provide guidance for clinical services and individual clinicians in virtual assessment and prevention of suicide risk. ${ }^{6}$

During a pandemic, screening questionnaires related to suicidal ideation can be considered in advance of a telephone or video visit. The National Alliance for Suicide 
Prevention in the US has prepared a guidance document for screening for suicide during virtual telehealth visits. ${ }^{7}$ When conducting virtual risk assessments, clinicians should be aware that clients may present in acute distress and should therefore obtain information about the client's exact location at the start of a virtual session. Clinicians should also determine alternate methods of connecting should technical interruptions occur during the session.

Smartphone and internet-based tools may potentially augment virtual suicide assessment and crisis management; however, current data about their efficacy are limited. Several recently developed phone apps appear to modestly reduce outcome metrics related to depression and self-harm but are not convincingly superior to control groups with respect to suicidal ideation. ${ }^{8,9}$ Self-guided internet-based tools, and in particular those which apply cognitive behavioural therapy, have been found to effectively reduce the risk of suicidal thoughts and behaviours when explicitly targeting suicidality. ${ }^{10,11}$ The accessibility and scalability of these resources provokes guarded optimism about their future in suicide assessment and prevention. However, in their current form, these tools' content and privacy protection are seldom regulated, and they are not equipped to provide multifaceted suicide prevention strategies (e.g., identifying risk factors/triggers or delivering psychological interventions) in the same way that clinicians can.

\section{Current evidence and future directions}

Although the effectiveness of virtual and tele-mental health interventions at reducing the risk of suicide and suicide-related behaviours has yet to be robustly compared to in-person services ${ }^{12}$, tele-mental health suicide assessments have been used for years by groups like the US Department of Veterans Affairs and telephone distress lines, and previous research has suggested that they are, in general, comparable to faceto-face assessments. ${ }^{13}$ The Collaborative Assessment and Management of Suicidality approach (an evidencebased therapeutic framework developed three decades ago for the assessment and management of patients at risk for suicide) has been promisingly adapted for delivery via tele-psychotherapy in recent work, although its effectiveness in reducing the risk of suicide and selfharm among adults remains understudied. ${ }^{14}$ Clinical experience suggests that virtual risk assessment may need to be modified, particularly with youth, patients with communication challenges, or patients with suspected psychotic symptoms. The authors have begun work to produce a practice guide for virtual and telehealth-based suicide risk assessment and crisis management.

Theory and research support the role of enhancing social outreach, connection, support, and perceptions of meaning in life and of mattering to others when seeking to reduce risk for suicide ideation and behaviour among older adults. Such efforts - including telephone support, older adult befriending programs, and meaningcentred group interventions - may be especially helpful in potentially counteracting the negative impact of epidemics on suicide risk in later life. ${ }^{15}$

Considering the evidence supporting the association between pandemics and both suicide and suiciderelated outcomes, government-funded suicide research and prevention initiatives are a priority at this time. Some critical gaps in our knowledge include ${ }^{1}$ :

- Are there particular populations (e.g., older adults, frontline healthcare workers, high population density/ urban dwellers, cultural minorities) who are at elevated risk of suicide-related outcomes during the COVID-19 pandemic compared with baseline rates? And, if so, are they amenable to targeted interventions?

- Are those directly exposed to the virus, or their families/caregivers, at elevated risk of suicide-related outcomes (whether immediately or over the longer term)?

- If rates of suicide-related outcomes change, what are the mechanisms or processes (e.g., neurobiological, psychological, social) that drive those changes?

- What is the trajectory of any observed changes (e.g., an initial decrease in suicide-related outcomes due to a 'pulling together' phenomenon followed by a steady increase versus an initial increase that slowly dissipates)?

- To what extent does the risk of suicide-related outcomes arise from factors unique to pandemics (e.g., social distancing or mass exposure to a virus with neuropsychiatric health sequelae) versus more general, ongoing risk factors (e.g., mental disorders, medical illness, access to means)?

- Can remote or virtual suicide risk assessments be conducted in a sensitive, safe, and effective fashion?

- What is the content quality of smartphone and internet-based assessment and intervention tools, and how can they most effectively be implemented at the clinical level?

The ongoing suicidogenic effect of the global COVID-19 pandemic, and of its distinct 'waves' and emerging viral strains like the Delta variant, remains yet to be fully understood. However, predictions based on previous pandemics suggest a heightened suicide risk for certain groups including older or dependent adults, and warrant conscientious innovation in the tools of suicide assessment. In particularly, the development of telemedicine, smartphone apps, and internet-based tools to identify and assess suicide risk - in combination with traditional approaches - will be valuable clinical priorities in the unique circumstances of the pandemic.

\section{Acknowledgements}

This project was supported by a grant from the Canadian Institutes of Health Research. 
The following team members contributed to the systematic literature review: Dr Giuseppe Guaiana, University of Western Ontario; Marisa Tippett, Western Libraries, University of Western Ontario; Nicole Edgar, Ottawa Hospital Research Institute; and Yevin Cha, Lawson Health Research Institute.

\section{Peer review and provenance}

Externally peer reviewed, not commissioned.

\section{Competing interests}

None declared.

\section{Author contributions}

All authors contributed to the manuscript's conception and the systematic literature review. CB and PL co-wrote the first draft of the manuscript and all authors contributed equally to editing and revising the first and subsequent drafts. All authors agreed on the final manuscript.

\section{References}

1. Zortea TC, Brenna CTA, Joyce M, McClelland H, Tippett M, Tran MM, et al. The impact of infectious disease-related public health emergencies on suicide, suicidal behavior, and suicidal thoughts. Crisis. 2020:114. Online ahead of print.

2. Pirkis J, John A, Shin S, DelPozo-Banos M, Arya V, Analuisa-Aguilar $P$, et al. Suicide trends in the early months of the COVID-19 pandemic: an interrupted timeseries analysis of preliminary data from 21 countries. Lancet Psychiatry. 2021;8(7):579-88

3. Carr MJ, Steeg S, Webb RT, Kapur N, Chew-Graham CA, Abel KM, et al. Effects of the COVID-19 pandemic on primary care-recorded mental illness and self-harm episodes in the UK: a population-based cohort study. Lancet Public Health. 2021;6(2):e124-35.

4. John A, Okolie C, Eyles E, Webb RT, Schmidt L, McGuiness LA, et al. The impact of the COVID-19 pandemic on self-harm and suicidal behaviour: a living systematic review. F1000Res. 2020;9:1097.
5. Stanley B, Brown GK. Telehealth tips: managing suicidal clients during the COVID-19 pandemic. Zero suicide. Massachusetts: Education Development Center; [cited 2021 Feb 1]. Available from: zerosuicide.edc.org/sites/ default/files/Telehealth\%20Tips\%20with\%20Suicidal\%20 Clients\%20-\%20FINAL.pdf

6. Galindo N. Assessing risk and preventing suicide via telehealth. North Carolina, US: RELIAS; 2020 [cited 2021 Feb 1]. Available from: www.relias.com/blog/assessingand-preventing-suicide-telehealth

7. National Action Alliance for Suicide Prevention. COVID guidance: screening for suicide during telehealth visits. Massachusetts, US: Action Alliance; 2020 [cited 2021 Feb 1]. Available from: theactionalliance.org/sites/default/files/ covid-suicidescreentelehealth_3.pdf

8. Melia R, Francis K, Hickey E, Bogue J, Duggan J, O'Sullivan M, Young K. Mobile health technology interventions for suicide prevention: systematic review. JMIR mHealth uHealth. 2020;8(1):e12516.

9. Arshad U, Gauntlett J, Husain N, Chaudhry N, Taylor PJ. A systematic review of the evidence supporting mobile-and internet-based psychological interventions for self-harm. Suicide Life-Threat Behav. 2020;50(1):151-79.

10. Büscher R, Torok M, Terhorst Y, Sander L. Internet-based cognitive behavioral therapy to reduce suicidal ideation: a systematic review and meta-analysis. JAMA Netw Open. 2020;3(4):e203933.

11. Torok M, Han J, Baker S, Werner-Seidler A, Wong I, Larsen ME, Christensen $\mathrm{H}$. Suicide prevention using self-guided digital interventions: a systematic review and meta-analysis of randomised controlled trials. Lancet Digit Health. 2020;2(1):e25-36.

12. Rojas SM, Carter SP, McGinn MM, Reger MA. A review of telemental health as a modality to deliver suicide-specific interventions for rural populations. Telemed J E Health. 2020;26(6):700-9.

13. Luxton DD, O'Brien K, Pruitt LD, Johnson K, Kramer G. Suicide risk management during clinical telepractice. Int J Psychiatry Med. 2014;48(1):19-31.

14. Hanratty D, Kilicaslan J, Wilding $H$, Castle D. A systematic review of efficacy of Collaborative Assessment and Management of Suicidality (CAMS) in managing suicide risk and deliberate self-harm in adult populations. Australas Psychiatry. 2019;27(6):559-64.

15. Heisel MJ, Moore SL, Flett GL, Norman RM, Links PS, Eynan R, et al. Meaning-centered men's groups: initial findings of an intervention to enhance resiliency and reduce suicide risk in men facing retirement. Clin Gerontol. 2020;43(1):76-94.

\section{Copyright: (c) (i) (2) (2)}

(C) 2021 Brenna et al. This article is licensed under the Creative Commons Attribution-NonCommercial-ShareAlike 4.0 International Licence, which allows others to redistribute, adapt and share this work non-commercially provided they attribute the work and any adapted version of it is distributed under the same Creative Commons licence terms. See: www.creativecommons.org/licenses/by-nc-sa/4.0/ 